\title{
Holographic thermalization with a chemical potential in Gauss-Bonnet gravity
}

\author{
Xiao-Xiong Zeng, ${ }^{a}$ Xian-Ming $\mathrm{Liu}^{b}$ and Wen-Biao Liu ${ }^{c}$ \\ ${ }^{a}$ School of Science, Chongqing Jiaotong University, \\ Chongqing, 400074, China \\ ${ }^{b}$ Department of Physics, Hubei University for Nationalities, \\ Enshi, 445000, Hubei, China \\ ${ }^{c}$ Department of Physics, Beijing Normal University, \\ Beijing, 100875, China \\ E-mail: xxzeng@mail.bnu.edu.cn, liuxianming1980@163.com, \\ wbliu@bnu. edu.cn
}

Abstract: Holographic thermalization is studied in the framework of Einstein-MaxwellGauss-Bonnet gravity. We use the two-point correlation function and expectation value of Wilson loop, which are dual to the renormalized geodesic length and minimal area surface in the bulk, to probe the thermalization. The numeric result shows that larger the Gauss-Bonnet coefficient is, shorter the thermalization time is, and larger the charge is, longer the thermalization time is, which implies that the Gauss-Bonnet coefficient can accelerate the thermalization while the charge has an opposite effect. In addition, we obtain the functions with respect to the thermalization time for both the thermalization probes at a fixed charge and Gauss-Bonnet coefficient, and on the basis of these functions, we obtain the thermalization velocity, which shows that the thermalization process is nonmonotonic. At the middle and later periods of the thermalization process, we find that there is a phase transition point, which divides the thermalization into an acceleration phase and a deceleration phase. We also study the effect of the charge and Gauss-Bonnet coefficient on the phase transition point.

KEYwORDs: AdS-CFT Correspondence, Black Holes

ARXIV EPRINT: 1311.0718 


\section{Contents}

1 Introduction 1

2 Charged Vaidya AdS black branes in Gauss-Bonnet gravity 3

3 Holographic thermalization $\quad \mathbf{5}$

3.1 Renormalized geodesic length 6

3.2 Minimal area surface 8

4 Numerical results $\quad 9$

5 Conclusions $\quad 18$

$\begin{array}{ll}\text { A Derivation of the Hamiltonian from the Lagrangian } & 19\end{array}$

\section{Introduction}

AdS/CFT correspondence [1] has been proved to be an effective tool to deal with the strong coupled systems. Especially in recent years, this correspondence has also been used extensively in non-equilibrium strong coupled systems [2-7]. One of the biggest motivation to use this gauge gravity duality to study the non-equilibrium strongly coupled systems is that the thermalization time of quark gluon plasma produced in RHIC and LHC experiments predicted by the perturbation theory is longer than the experiment result. The reason for this difference is that the perturbation theory [8] treats the thermalization process as a near-equilibrium process, where the static black hole in the bulk is dual to the boundary system in equilibrium with finite temperature. However, the sudden injection of energy in the thermalization process is a far-from-equilibrium behavior of strongly coupled systems, the holographic bulk thus should be a highly dynamical spacetime, which can be described as black hole formation or black hole merger. Based on this paradigm, there have been many models to study the far-from-equilibrium thermalization behaviors [9-17] recently. Especially in $[18,19]$, Balasubramanian et al. find that one can use the two-point correlation function, Wilson loop, and entanglement entropy, which can further be evaluated in the saddle point approximation in terms of geodesic, minimal surface, and minimal volume individually, to detect the thermalization, where the initial state in the conformal field theory is dual to the AdS boundary in a higher dimensional space time, the sudden injection of energy is dual to the collapse of a thin shell of dust, and the final equilibrium state is dual to a static black brane. It is found that the holographic thermalization always proceeds in a top-down pattern, namely the UV modes thermalize firstly, followed by the 
IR modes. ${ }^{1}$ They also find that there is a slight delay in the onset of thermalization and the entanglement entropy thermalizes slowest, which sets a timescale for equilibration. Later, such an investigation is generalized to the bulk geometry with electrostatic potential [20$22]$ and high curvature corrections [23-25] to see how the chemical potential and correction parameter affect the thermalization time in the boundary field theory, other extensions on this topic please see [26-29]. Made by Balasubramanian et al., there are further some elegant extensions on holographic thermalization very recently. Firstly the time-dependent spectral functions in conformal field is found to be tractable [30]. With it, many quantities of interest in the thermalization process, e.g., the time dependence of the occupation number of field modes in the boundary gauge theory, can be calculated. Secondly, the holographic thermalization is extended to the inhomogeneous case by considering an $A d S_{4}$ weak field inhomogeneous collapse $[31,32]$. It is found that the AdS description of the early time evolution is well-matched by free streaming, and the stress tensor approaches that of second order hydrodynamics near the end of the early time interval.

The purpose of this paper is to investigate holographic thermalization in the bulk with curvature corrections and a gauge potential. By holography, curvature correction corresponds to $\frac{1}{N}$ or $\frac{1}{\lambda}$ correction $[33,34]$ to the boundary field theory, ${ }^{2}$ and the gauge potential corresponds to a chemical potential in the boundary field theory. In Einstein gravity, there have been some works to study the effect of the chemical potential on the thermalization $[20,21]$. And there are also some works to study the effect of correction parameter of the high order curvature on the thermalization [23-25]. Here we want to explore whether the chemical potential and the correction parameter have the same effect on the thermalization time. If not, our model will provide theoretically a wider range of the thermalization time. To observe the thermalization process in the dual conformal field theory, we take the two-point correlation function and expectation value of Wilson loop as thermalization probes to study the thermalization behavior. ${ }^{3}$ According to the AdS/CFT correspondence, this process equals to probing the evolution of a shell of charged dust that interpolates between a pure AdS and a charged Gauss-Bonnet AdS black brane by making use of the renormalized geodesic length and minimal area surface. Concretely we first study the motion profile of the geodesic and minimal area, and then the renormalized geodesic length and minimal area surface in the charged Gauss-Bonnet Vaidya AdS black brane. When we study the effect of the gauge potential on the thermalization process,

\footnotetext{
${ }^{1}$ On the weakly coupled side classical calculations have shown that the thermalization process is of the bottom-up type, i.e. low energetic modes reach thermal equilibrium first [8].

${ }^{2}$ According to the AdS/CFT correspondence, it is well known that IIB string theory on AdS5 $\times S^{5}$ background is dual to $D=4 N=4 \mathrm{SU}\left(N_{c}\right)$ super Yang-Mills theory. In the limits of large $N_{c}$ and large 't Hooft coupling, the SYM theory is dual to IIb supergravity which is low energy effective theory of superstring theory. So the higher curvature terms in the bulk theory can arise as next to leading order corrections in the $1 / N($ large $N$ ) expansion of the boundary CFTs in the strong 't Hooft coupling limit.

${ }^{3}$ Usually, one also can use the entanglement entropy to detect the thermalization process. Recently there have been many works to study holographic entanglement entropy in higher derivative gravitational theories [35-41]. Especially in [25], the author found that the entanglement entropy has similar behavior as the other observables during the thermalization process. For simplicity, we will not study entanglement entropy in this paper.
} 
the Gauss-Bonnet coefficient is fixed and when we are interested in the effect of GaussBonnet coefficient, the gauge potential is fixed. Our result shows that larger the GaussBonnet coefficient is, easier the dual boundary system thermalizes, while larger the charge is, harder the dual boundary system thermalizes. That is, the Gauss-Bonnet coefficient have an opposite effect on the thermalization time compared with the gauge potential. In addition, we also obtain the analytical functions of the renormalized geodesic length with respect to the thermalization time as well as the renormalized minimal area surface with respect to the thermalization time. Based on the functions, we get the thermalization velocity. Our result shows that the velocity is negative at the initial time and positive at the middle and later periods, which indicates that the thermalization process is nonmonotonic. We find that there is a phase transition point for the thermalization velocity at a fixed $Q$ and $\alpha$, which divides the thermalization process into an acceleration phase and a deceleration phase. Effect of $Q$ and $\alpha$ on the phase transition points is also studied.

The remainder of this paper is organized as follows. In the next section, we shall provide a brief review of the charged Vaidya AdS black brane in Gauss-Bonnet gravity. Then the holographic setup for non-local observables will be explicitly constructed in section 3 . Especially, we show that the relation between the renormalized length and the two-point correlation function is still valid in the Gauss-Bonnet gravity. Resorting to numerical calculation, we perform a systematic analysis of how the Gauss-Bonnet coefficient and chemical potential affect the thermalization time and thermalization velocity in section 4 . We end up with some discussions in the last section.

\section{Charged Vaidya AdS black branes in Gauss-Bonnet gravity}

The $D(D \geq 5)$ dimensional Einstein-Maxwell theory with a negative cosmological constant and a Gauss-Bonnet term ${ }^{4}$ representing a quadratic curvature correction is given by

$$
I=\frac{1}{16 \pi G_{D}} \int_{\mathcal{M}} d^{D} x \sqrt{-g}\left(R-2 \Lambda-4 \pi G_{D} F_{\mu \nu} F^{\mu \nu}+\alpha L_{G B}\right),
$$

where $G_{D}$ is the D-dimensional gravitational constant, $R$ is the Ricci scalar, $\Lambda$ is the negative cosmological constant, $\alpha$ is the Gauss-Bonnet coefficient, and $F_{\mu \nu}=\partial_{\mu} A_{\nu}-\partial_{\nu} A_{\mu}$,

$$
L_{G B}=R^{2}-4 R_{\mu \nu} R^{\mu \nu}+R_{\mu \nu \sigma \tau} R^{\mu \nu \sigma \tau} .
$$

From the action in eq. (2.1), many exact solutions have been found [43-50], here we are interested in the D-dimensional charged black brane solution

$$
d s^{2}=-F(r) d t^{2}+F(r)^{-1} d r^{2}+\frac{r^{2}}{l^{2}} d x_{n}^{2}
$$

where

$$
F(r)=\frac{r^{2}}{2 \tilde{\alpha}}\left[1-\sqrt{1-\frac{4 \tilde{\alpha}}{l^{2}}\left(1-\frac{M l^{2}}{r^{D-1}}+\frac{Q^{2} l^{2}}{r^{2 D-4}}\right)}\right],
$$

\footnotetext{
${ }^{4}$ The action in eq. (2.1) is not a consistent truncation of an effective higher derivative gravitational action, it is one special case where the higher order corrections of the Maxwell field have vanishing coefficients. One also can take into account the corrections to the Maxwell field such as in [42, 43].
} 
in which $\ell=\sqrt{-\frac{(D-1)(D-2)}{2 \Lambda}}, \tilde{\alpha}=(D-3)(D-4) \alpha, \mathrm{M}$ and $\mathrm{Q}$ are related to the ADM black hole mass $M_{0}$ and charge $Q_{0}$ as follows

$$
\begin{aligned}
& M_{0}=\frac{(D-2) M V_{n}}{16 \pi G_{D}}, \\
& Q_{0}^{2}=\frac{2 \pi(D-2)(D-3) Q^{2}}{G_{D}},
\end{aligned}
$$

where $V_{n}$ is the volume of the unit radius sphere $S^{D-2}$. The $\mathrm{U}(1)$ gauge potential reads

$$
A_{t}=-\frac{Q}{4 \pi(D-3)}\left(r_{h}^{3-D}-r^{3-D}\right)=u\left(1-\frac{r_{h}^{D-3}}{r^{D-3}}\right),
$$

in which $r_{h}$ is the event horizon radius that is characterized by $F\left(r_{h}\right)=0$ and $u$ is the electrostatic potential, which can be identified with the chemical potential of the dual field theory. The Hawking temperature of the charged Gauss-Bonnet AdS black brane reads

$$
T=\left.\frac{\partial_{r} F(r)}{4 \pi}\right|_{r_{h}}=\frac{(D-1) r_{h}-(D-3) Q^{2} \ell^{2} r_{h}^{5-2 D}}{4 \pi \ell^{2}},
$$

which can be viewed as the temperature of the dual conformal field theory on the AdS boundary. On the other hand, as $r$ approaches to infinity, one can see the above black brane metric changes into

$$
d s^{2} \rightarrow \frac{r^{2}}{\ell_{\mathrm{eff}}^{2}}\left(-d t^{2}+d \tilde{x}_{n}^{2}\right)+\frac{\ell_{\mathrm{eff}}^{2}}{r^{2}} d r^{2}
$$

where

$$
\tilde{x}_{n}=\frac{\ell_{\mathrm{eff}}}{\ell} x_{n}, \quad \ell_{\mathrm{eff}}^{2}=\frac{2 \tilde{\alpha}}{1-\sqrt{1-\frac{4 \tilde{\alpha}}{\ell^{2}}}} .
$$

Thus this black brane solution is asymptotically AdS with AdS radius $\ell_{\text {eff }}$.

To get a Vaidya type evolving black brane, we would like first to make the coordinate transformation $z=\frac{\ell^{2}}{r}$, in this case the black brane metric in eq. (2.3) can be cast into

$$
d s^{2}=\frac{\ell^{2}}{z^{2}}\left[-F(z) d t^{2}+F^{-1}(z) d z^{2}+d x_{n}^{2}\right]
$$

where

$$
F(z)=\frac{\ell^{2}}{2 \tilde{\alpha}}\left[1-\sqrt{1-\frac{4 \tilde{\alpha}}{\ell^{2}}\left(1-M z^{D-1} \ell^{4-2 D}+Q^{2} z^{2 D-4} \ell^{10-4 D}\right)}\right] .
$$

Then by introducing the Eddington-Finkelstein coordinate system, namely

$$
d v=d t-\frac{1}{F(z)} d z
$$

eq. (2.10) changes into

$$
d s^{2}=\frac{\ell^{2}}{z^{2}}\left[-F(z) d v^{2}-2 d z d v+d x_{n}^{2}\right]
$$


Now the charged Gauss-Bonnet Vaidya AdS black brane can be obtained by freeing the mass parameter and charge parameter as arbitrary functions of $v$ [51-53]. This black brane is a solution of the equations of motion of the total action

$$
I_{\text {total }}=I+I_{\text {matter }}
$$

where $I_{\text {matter }}$ is the action for external matter fields. As one can show, such a metric is sourced by the null dust with the energy momentum tensor flux and gauge flux [51-53]

$$
\begin{aligned}
& 8 \pi G_{D} T_{\mu \nu}^{\text {matter }}=z^{D-2}\left(\frac{D-2}{2} \dot{M}(v)-(D-2) z^{D-3} Q(v) \dot{Q}(v)\right) \delta_{\mu v} \delta_{\nu v}, \\
& 8 \pi G_{D} J_{\text {matter }}^{\mu}=\sqrt{\frac{(D-2)(D-3)}{2}} z^{D} \dot{Q}(v) \delta^{\mu z}
\end{aligned}
$$

where the dot stands for derivative with respect to coordinate $v, M(v)$ and $Q(v)$ are the mass and charge of a collapsing black brane. ${ }^{5}$ It is obvious that for the charged Vaidya AdS black branes in Gauss-Bonnet gravity, the energy-momentum tensor depends on not only $M(v)$ but also $Q(v)$. In this case, eq. (2.13) describes the collapse of a thin-shell of charged dust from the boundary toward the bulk interior of asymptotically anti-de Sitter spaces as $M$ and $Q$ are substituted by $M(v)$ and $Q(v)$.

\section{Holographic thermalization}

In this section, we are going to investigate the thermalization process of a class of strongly coupled system. According to the AdS/CFT correspondence, the rapid injection of energy on the boundary corresponds to the collapse of a black brane in the AdS space. So to describe the thermalization process holographically, one should choose the mass $M(v)$ and charge $Q(v)$ properly so that it can describe the evolution of the charged dust. It was found that this properties can be achieved by setting the mass parameter and charge parameter as $M(v)=M \theta(v), Q(v)=Q \theta(v)[20,21]$, where $\theta(v)$ is the step function. In this case, in the limit $v \rightarrow-\infty$, the background corresponds to a pure AdS space while in the limit $v \rightarrow \infty$, it corresponds to a charged Gauss-Bonnet AdS black brane. For the convenience of numerical calculations, $M(v)$ and $Q(v)$ are usually chosen as the smooth functions

$$
\begin{aligned}
M(v) & =\frac{M}{2}\left(1+\tanh \frac{v}{v_{0}}\right), \\
Q(v) & =\frac{Q}{2}\left(1+\tanh \frac{v}{v_{0}}\right),
\end{aligned}
$$

where $v_{0}$ represents a finite shell thickness.

Having the construction of a model that describes the thermalization process on the dual conformal field theory, we have to choose a set of extended non-local observables ${ }^{6}$ in

\footnotetext{
${ }^{5}$ For more information about the energy condition in the time dependent background, please see [54].

${ }^{6}$ Because the local observables such as the energy-momentum tensor and its derivatives can not explore deviations from thermal equilibrium in detail though it provides valuable information about the applicability of viscous hydrodynamics.
} 
the bulk which allow us to evaluate the evolution of the system. In this paper, we shall focus mainly on the two-point correlation function at equal time and expectation value of rectangular space-like Wilson loop, which in the bulk correspond to the renormalized geodesic length and minimal area surface respectively. For simplicity but without loss of generality, we shall set the unit such that $\ell=1$ and $r_{h}=1$ in the later discussions. In addition, from eq. (2.11), we know that the mass and the charge have the relation $M=1+Q^{2}$, in this case we only need to change the charge to adjust the mass parameter during the numerical process.

\subsection{Renormalized geodesic length}

The relation between the renormalized geodesic length and two-point correlation function at equal time has been discussed extensively $[19,55]$. Here we will give a review of the relation to check whether it is still valid in the Gauss-Bonnet gravity.

Start with the action of a complex scalar field of mass $m$ in the bulk background gravitational field $g_{a b}$

$$
S=-\frac{1}{2} \int d^{d+1} X \sqrt{-g}\left[g^{a b} \nabla_{a} \bar{\phi} \nabla_{b} \phi+m^{2} \bar{\phi} \phi\right],
$$

which gives rise to the bulk propagator

$$
G(x, y)=\langle\bar{\phi}(x) \phi(y)\rangle=\left\langle x\left|\frac{1}{i H}\right|\right\rangle y=\int_{0}^{\infty} d T\left\langle x\left|e^{-i H T}\right| y\right\rangle,
$$

where $H=-\frac{1}{2}\left[\nabla_{a} \nabla^{a}-m^{2}\right]$ can be thought of as the Hamiltonian of a fictitious quantum mechanical model with $T$ the proper time. ${ }^{7}$ With this in mind, one can reformulate this quantum mechanical model in terms of path integral over the worldlines of a massive particle. In particular, the above bulk propagator can be expressed as

$$
\begin{aligned}
G(x, y) & =\int_{0}^{\infty} d T \mathcal{D} X e^{i s} \\
& =\mathcal{N} \int_{0}^{\infty} d T \prod_{\tau} \int D X(\tau) \sqrt{-g(\tau)} e^{i \int_{0}^{T} d \tau\left[\frac{1}{2}\left(g_{\mu \nu} \frac{d X^{\mu}}{d \tau} \frac{d X^{\nu}}{d \tau}-m^{2}\right)+\frac{R}{6}\right]} \\
& =\mathcal{N} \int_{0}^{\infty} d T \prod_{\tau} \int D X(\tau) \sqrt{-g(\tau)} e^{i \int_{0}^{1} d \tau\left[\frac{1}{2}\left(T^{-1} g_{\mu \nu} \frac{d X^{\mu}}{d \tau} \frac{d X^{\nu}}{d \tau}-T \hat{m}^{2}\right)\right]}
\end{aligned}
$$

with $X(0)=y, X(T)=x$, and $\hat{m}^{2}=m^{2}-\frac{R}{3}$. In the saddle point approximation, i.e.,

$$
T=\frac{\sqrt{-g_{\mu \nu} \frac{d X^{\mu}}{d \tau} \frac{d X^{\nu}}{d \tau}}}{\hat{m}},
$$

the bulk propagator can be evaluated as

$$
G(x, y) \propto e^{i \int_{0}^{1} d \tau\left(-\hat{m} \sqrt{-g_{\mu \nu} \frac{d X^{\mu}}{d \tau} \frac{d X^{\nu}}{d \tau}}\right)},
$$

\footnotetext{
${ }^{7}$ For the derivation of the Hamiltonian, please see appendix.
} 
with $X^{\mu}(\tau)$ the classical trajectory satisfying the above equation of motion. Obviously eq. (3.7) is consistent with the formulation in $[19,55]$

$$
\left\langle\mathcal{O}\left(t_{0}, \mathbf{x}\right) \mathcal{O}\left(t_{0}, \mathbf{x}^{\prime}\right)\right\rangle \approx e^{-\Delta \tilde{L}_{\mathrm{ren}}},
$$

where $\Delta$ is the conformal dimension of scalar operator $\mathcal{O}$, which is similar to $\hat{m}$ in eq. (3.7), and $\tilde{L}_{\text {ren }}$ indicates the renormalized length of the bulk geodesic between the points $\left(t_{0}, x_{n}\right)$ and $\left(t_{0}, x_{n}^{\prime}\right)$ on the AdS boundary. In other words, the Gauss-Bonnet coefficient has not effect on the dual relation between the renormalized geodesic length and the two-point correlation function. In what follows, we will make use of eq. (3.8) to explore how the Gauss-Bonnet coefficient and gauge potential affect the thermalization time. The reason that we use the renormalized geodesic length $\tilde{L}_{\text {ren }}$ in eq. (3.7) is that the geodesic length is divergent near the boundary, which is related to the divergent part as

$$
\tilde{L}_{\text {ren }}=\tilde{L}+2 \ell_{\text {eff }} \ln z_{0} .
$$

where $\tilde{L}=\sqrt{-d S^{2}}$ is the geodesic length between the points $\left(t_{0}, x_{n}\right)$ and $\left(t_{0}, x_{n}^{\prime}\right)$ on the AdS boundary and $z_{0}$ is the IR radial cut-off. Next, we are concentrating on studying $\tilde{L}$. Taking into account the spacetime symmetry of our Vaidya type black brane, we can simply let $\left(t_{0}, x_{n}\right)$ and $\left(t_{0}, x_{n}^{\prime}\right)$ have identical coordinates except $x_{1}=-\ell_{\text {eff }} \frac{l}{2} \equiv-\frac{\tilde{l}}{2}$ and $x_{1}^{\prime}=-\ell_{\mathrm{eff}} \frac{l}{2} \equiv-\frac{\tilde{l}}{2}$ with $\tilde{l}$ the separation between these two points on the boundary. In order to make the notation as simple as possible, we would like to rename this exceptional coordinate $x_{1}$ as $x$ and employ it to parameterize the trajectory such that the proper length in the charged Gauss-Bonnet Vaidya AdS black brane can be given by

$$
\tilde{L}=\int_{-\frac{\tilde{l}}{2}}^{\frac{\tilde{\tau}}{2}} d x \frac{\sqrt{1-2 z^{\prime}(x) v^{\prime}(x)-F(v, z) v^{\prime}(x)^{2}}}{z(x)},
$$

where the prime denotes the derivative with respect to $x$ and

$$
F(v, z)=\frac{1}{2 \tilde{\alpha}}\left[1-\sqrt{1-4 \tilde{\alpha}\left(1-M(v) z^{D-1}+Q(v)^{2} z^{2 D-4}\right)}\right] .
$$

Note that the integrand in eq. (3.10) can be thought of as the Lagrangian $\mathcal{L}$ of a fictitious system with $x$ the proper time. Since the Lagrangian does not depend explicitly on $x$, there is an associated conserved quantity

$$
\mathcal{H}=\mathcal{L}-v^{\prime}(x) \frac{\partial \mathcal{L}}{\partial v^{\prime}(x)}-z^{\prime}(x) \frac{\partial \mathcal{L}}{\partial z^{\prime}(x)}=\frac{1}{z(x) \sqrt{1-2 z^{\prime}(x) v^{\prime}(x)-F(v, z) v^{\prime}(x)^{2}}} .
$$

In addition, based on the Lagrangian $\mathcal{L}$ of the system, we obtain

$$
\begin{aligned}
\frac{\partial \mathcal{L}}{\partial z} & =\frac{-v^{\prime}(x)^{2} \partial_{z} F(z, v) \mathcal{H}}{2}-\frac{1}{z(x)^{3} \mathcal{H}}, \\
\frac{\partial}{\partial x} \frac{\partial \mathcal{L}}{\partial z^{\prime}} & =-v^{\prime \prime}(x) \mathcal{H} .
\end{aligned}
$$

So the equation of motion for $z(x)$ can be written as

$$
0=2-2 v^{\prime}(x)^{2} F(v, z)-4 v^{\prime}(x) z^{\prime}(x)-2 z(x) v^{\prime \prime}(x)+z(x) v^{\prime}(x)^{2} \partial_{z} F(v, z) .
$$


Similarly, the equation of motion for $v(x)$ can be solved as

$$
0=v^{\prime}(x) z^{\prime}(x) \partial_{z} F(v, z)+\frac{1}{2} v^{\prime}(x)^{2} \partial_{v} F(v, z)+v^{\prime \prime}(x) F(v, z)+z^{\prime \prime}(x),
$$

in which

$$
\begin{aligned}
& \partial_{z} F(z, v)=\frac{(D-1) M(v) z^{D-2}-(2 D-4) Q^{2}(v) z^{2 D-5}}{\sqrt{1-4 \tilde{\alpha}\left(1-M(v) z^{D-1}+Q^{2}(v) z^{2 D-4}\right)}}, \\
& \partial_{v} F(z, v)=\frac{M^{\prime}(v) z^{D-1}-2 Q(v) Q^{\prime}(v) z^{2 D-4}}{\sqrt{1-4 \tilde{\alpha}\left(1-M(v) z^{D-1}+Q^{2}(v) z^{2 D-4}\right)}} .
\end{aligned}
$$

Next, we turn to studying the equations of motion in eq. (3.15) and eq. (3.16). Considering the reflection symmetry of our geodesic, we will use the following initial conditions

$$
z(0)=z_{*}, v(0)=v_{*}, v^{\prime}(0)=z^{\prime}(0)=0 .
$$

As $z(x)$ and $v(x)$ are solved, we can get the IR radial cut-off and thermalization time by the boundary conditions as follows

$$
z\left(\frac{\tilde{l}}{2}\right)=z_{0}, v\left(\frac{\tilde{l}}{2}\right)=t_{0}
$$

In addition, with the help of eq. (3.12) and eq. (3.18), the proper length of geodesic in eq. (3.10) can be simplified as

$$
\tilde{L}=2 \int_{0}^{\frac{\tilde{l}}{2}} d x \frac{z_{*}}{z(x)^{2}}
$$

which will be more convenient for us to obtain the renormalized geodesic length.

\subsection{Minimal area surface}

In this section, we are going to study the minimal area surface, which in the dual conformal field theory corresponds to the Wilson loop operator. Wilson loop operator is defined as a path ordered integral of gauge field over a closed contour, and its expectation value is approximated geometrically by the AdS/CFT correspondence as $[19,56]$

$$
\langle W(C)\rangle \approx e^{-\frac{\tilde{A}_{\mathrm{ren}}(\Sigma)}{2 \pi \alpha^{\prime}}},
$$

where $C$ is the closed contour, $\Sigma$ is the minimal bulk surface ending on $C$ with $\tilde{A}_{\text {ren }}$ its renormalized minimal area surface, and $\alpha^{\prime}$ is the Regge slope parameter. In the GaussBonnet gravity, we will assume eq. (3.21) is still valid as that between the renormalized geodesic length and the two-point correlation function.

Here we are focusing solely on the rectangular space-like Wilson loop. In this case, the enclosed rectangle can always be chosen to be centered at the coordinate origin and lying on the $x_{1}-x_{2}$ plane with the assumption that the corresponding bulk surface is invariant along the $x_{2}$ direction. This implies that the minimal area surface can be expressed as

$$
\tilde{A}=\int_{\frac{\tilde{l}}{2}}^{\frac{\tilde{l}}{2}} d x \frac{\sqrt{1-2 z^{\prime}(x) v^{\prime}(x)-F(v, z) v^{\prime}(x)^{2}}}{z(x)^{2}},
$$


where we have set the separation along $x_{2}$ direction to be one and the separation along $x_{1}$ to be $\tilde{l}$ with $x_{2}$ renamed as $y$ and $x_{1}$ renamed as $x$. As before, from eq. (3.22) we also can get a Lagrangian $\mathcal{L}$ and with it we can find a conserved quantity, i.e.,

$$
\mathcal{H}=\frac{1}{z(x)^{2} \sqrt{1-2 z^{\prime}(x) v^{\prime}(x)-F(v, z) v^{\prime}(x)^{2}}},
$$

which can simplify our equations of motion as

$$
\begin{aligned}
& 0=4-4 v^{\prime}(x)^{2} F(v, z)-8 v^{\prime}(x) z^{\prime}(x)-2 z(x) v^{\prime \prime}(x)+z(x) v^{\prime}(x)^{2} \partial_{z} F(v, z), \\
& 0=v^{\prime}(x) z^{\prime}(x) \partial_{z} F(v, z)+\frac{1}{2} v^{\prime}(x)^{2} \partial_{v} F(v, z)+v^{\prime \prime}(x) F(v, z)+z^{\prime \prime}(x) .
\end{aligned}
$$

Similarly, with the initial conditions as in (3.18) and the regularization cut-off as in (3.19), the renormalized minimal area surface can be cast into

$$
\tilde{A}_{\mathrm{ren}}=2 \int_{0}^{\frac{\tilde{\tau}}{2}} d x \frac{z_{*}^{2}}{z(x)^{4}}-\frac{2}{z_{0}} .
$$

Next, we will investigate the evolution of the renormalized minimal area surface with respect to the thermalization time to explore how the gauge potential and Gauss-Bonnet coefficient affect the thermalization process.

\section{Numerical results}

In this section, we will solve the equations of motion of geodesic length and minimal area surface numerically, and then explore how the chemical potential and Gauss-Bonnet coefficient affect the thermalization time. Since there have been many works to study the effect of the space time dimensions and boundary separation on the thermalization probes [18-21], to avoid redundancy, we mainly discuss the case $D=5$ and a fixed boundary separation in this paper. During the numerics, we will take the shell thickness and UV cut-off as $v_{0}=0.01, z_{0}=0.01$ respectively.

According to the AdS/CFT correspondence, we know that the electromagnetic field in the bulk is dual to the chemical potential in the dual quantum field theory, so we will use the electromagnetic field defined in eq. (2.6) to explore the effect of the chemical potential on the thermalization process in the AdS boundary. However, as stressed in $[20,57,58]$, the chemical potential has energy units in the dual field theory $([u]=1 /[L])$ while $A_{\mu}$ as defined in eq. (2.1) is dimensionless, thus one has to redefine the electromagnetic field as $\tilde{A}_{\mu}=$ $A_{\mu} / p$, where $p$ is a scale with length unit that depends on the particular compactification. In this case, $\tilde{A}_{\mu}$ and $u$ have the same unit and the chemical potential can be expressed as

$$
u=\lim _{r \rightarrow \infty} \tilde{A}_{\mu}=\frac{Q r_{h}^{3-D}}{4 \pi p(D-3)} .
$$

In addition, due to the conformal symmetry on the boundary, the quantity which is physically meaningful is the ratio of $u / T$ in asymptotically charged AdS space time, namely 
the chemical potential measured with the temperature as the unit. Therefore, through this paper we will use the following ratio

$$
\frac{u}{T}=\frac{Q}{p(D-3)\left[(D-1) r_{h}^{D-2}-(D-3) Q^{2} r_{h}^{3-D}\right]},
$$

to check the effect of the chemical potential on the thermalization time. Obviously, for the case $r_{h}=1$, eq. (4.2) shows that $u / T$ changes from $0 \rightarrow \infty$ provided $Q$ changes from $0 \rightarrow \sqrt{\frac{D-1}{D-3}}$. In other words, to adjust the change of the ratio $u / T$ in all the range, we only need to change $Q$ from $0 \rightarrow \sqrt{\frac{D-1}{D-3}}$. For the case $D=5$ in this paper, we will choose $Q=0.00001,0.5,1$ in our numerical result. On the other hand, we will also consider the effect of the Gauss-Bonnet coefficient on the thermalization time, as in [23] we will take $\alpha=-0.1,0.0001,0.08$ as the constraint of causality of dual field theory on the boundary is imposed. ${ }^{8}$

As the boundary conditions in eq. (3.18) is adopted, the equations of motion of the geodesic in eq. (3.15) and eq. (3.16) for different $\alpha$ and different $Q$ can be solved numerically. When we are interested in the effect of $Q$ on the motion profile of the geodesic, the GaussBonnet coefficient $\alpha$ is fixed, and when we are interested in the effect of Gauss-Bonnet coefficient $\alpha$, the charge $Q$ is fixed. Since different initial time $v_{\star}$ corresponds to different stage of the motion of the geodesics, we also discuss the effect of $v_{\star}$ on the motion profile. Figure 1 and figure 2 plot the motion profile of the geodesic at the initial time $v_{\star}=$ $-0.856,-0.456$ respectively for different charge and Gauss-Bonnet coefficient. In both figures, the horizontal direction is the motion profile of the geodesics for different GaussBonnet coefficients while the vertical direction is the motion profile of the geodesics for different charges. From figure 1 and figure 2, we know that as the initial time increases, the shell of the dust approaches to the horizon of the charged Gauss-Bonnet black brane, which means that for the larger initial time, the thermalization has been behaved longer. For different initial time, we also keep a watchful eye on how the Gauss-Bonnet coefficient and charge affect the thermalization time, which are listed in table (1). From this table, we can observe that for both the two different initial times, as the Gauss-Bonnet coefficient grows, the thermalization time decreases, which means that the quark gluon plasma in the dual conformal theory is easier to be thermalized. But as the charge grows, we find for different initial times, the thermalization time has different variation trends. For the case $v_{\star}=-0.856$, it is found that for a fixed Gauss-Bonnet coefficient, as the charge grows the thermalization time has a little difference, while for $v_{\star}=-0.456$, the thermalization time increases as the charge grows. That is, the charge has little effect on the thermalization time at the initial stage of the thermalization. We also can observe the effect of the GaussBonnet coefficient and charge on the motion profile of the geodesics. From figure 2, we find for a fixed charge, e.g. $Q=0.5$, as the Gauss-Bonnet coefficient grows from $\alpha=-0.1$ to $\alpha=0.08$, the shell will approaches to the horizon of the black brane and drops into the horizon lastly. In other words, for $\alpha=-0.1$, the quark gluon plasma in the conformal

\footnotetext{
${ }^{8} \mathrm{As} \alpha$ is dialed beyond the causality bound but within the Chern-Simons limit, it has not effect on the thermalization process. One can see the thermalization curves plotted in [25].
} 
field theory is thermalizing while for $\alpha=0.08$, it is thermalized. For a fixed Gauss-Bonnet coefficient, e.g. $\alpha=0.08$, we find at $Q=0.00001$, the shell lies above the horizon while at $Q=1$, the shell lies below the horizon.

Having the numerical result of $z(x)$, we can study the renormalized geodesic length with the help of eq. (3.9) and eq. (3.20). As done in [20], we compare $\delta \tilde{L}$ at each time with the final values $\delta \tilde{L}_{C G B}$, obtained in a static charged Gauss-Bonnet AdS black brane, i.e. $M(\mu)=M, Q(\mu)=Q$. In this case, the thermalized state is labeled by the zero point of the vertical coordinate in each picture. To get an observable quantity that is $\tilde{l}$ independent, we will plot the quantity $\delta L=\delta \tilde{L} / \tilde{l}$. Figure 3 gives the relation between the renormalized geodesic length and thermalization time for different charge at a fixed Gauss-Bonnet coefficient. In each picture, the vertical axis indicates the renormalized geodesic length while the horizontal axis indicates the thermalization time $t_{0}$. For a fixed Gauss-Bonnet coefficient, e.g. $\alpha=0.08$, the thermalization time increases as $Q$ raises. This phenomenon has been also observed previously when we study the motion profile of the geodesic. In [20], the effect of charge on the thermalization time is investigated in Einstein gravity, it was shown that there is an enhancement of the thermalization time as the ratio of chemical potential over temperature increases. Obviously, in the Gauss-Bonnet gravity, this phenomenon is not changed. In addition, From the same color line, e.g. green line, in (a), (b) and (c) in figure 3, we know that as the Gauss-Bonnet coefficient increases, the thermalization time decreases for a fixed charge. In figure 4, we plot this graphics for different charge. Note that [23] has investigated this phenomenon for the case $Q=0$. It was found that for a fixed boundary separation there is always a time range in which the renormalized geodesic length takes the same value nearly. That is, during that time range, the Gauss-Bonnet coefficient has little effect on the renormalized geodesic length. Obviously, (a) in figure 4 is consistent with their result. For $Q=0.5$ and $Q=1$, we also can observe this phenomenon, which are plotted in (b) and (c) in figure 4.

Interestingly, we find the thermalization curve for a fixed charge and Gauss-Bonnet coefficient in figure 3 can be fitted as a function of $t_{0}$. Figure 5 is the comparison result of the numerical curve and function curve. At a fixed $\alpha$, one can get the function of the thermalization curve for different charge. For example, at $\alpha=0.0001$, the thermalization curve for $Q=0.00001,0.5,1$ can be expressed respectively as ${ }^{9}$

$$
\left\{\begin{array}{c}
g_{1}=-0.132319-0.015338 t_{0}+0.132916 t_{0}^{2}-0.393653 t_{0}^{3} \\
+0.560646 t_{0}^{4}-0.190989 t_{0}^{5}-0.0210284 t_{0}^{6} \\
g_{2}=-0.140525-0.0101622 t_{0}+0.101997 t_{0}^{2}-0.348701 t_{0}^{3} \\
+0.626571 t_{0}^{4}-0.324671 t_{0}^{5}+0.033151 t_{0}^{6} \\
g_{3}=-0.166815-0.00146773 t_{0}+0.0355258 t_{0}^{2}-0.209675 t_{0}^{3} \\
+0.691328 t_{0}^{4}-0.54898 t_{0}^{5}+0.129204 t_{0}^{6}
\end{array}\right.
$$

For small time, the function is determined by the lower power of $t_{0}$, while for large time it is determined by the higher power of $t_{0}$. With the function, we can get the thermalization

\footnotetext{
${ }^{9}$ For higher order power of $t_{0}$, we find it has few contributions to the thermalization, including the phase transition point which will be discussed next.
} 


\begin{tabular}{|l|c|c|c|c|c|c|}
\hline & \multicolumn{3}{|c|}{$v_{\star}=-0.856$} & \multicolumn{3}{c|}{$v_{\star}=-0.456$} \\
\hline & $\alpha=-0.1$ & $\alpha=0.0001$ & $\alpha=0.08$ & $\alpha=-0.1$ & $\alpha=0.0001$ & $\alpha=0.08$ \\
\hline$Q=0.00001$ & 0.691064 & 0.625561 & 0.560275 & 1.01538 & 0.949617 & 0.887499 \\
\hline$Q=0.5$ & 0.690223 & 0.624786 & 0.559595 & 1.02213 & 0.958193 & 0.897744 \\
\hline$Q=1$ & 0.687522 & 0.622449 & 0.557604 & 1.03968 & 0.981075 & 0.925744 \\
\hline
\end{tabular}

Table 1. The thermalization time $t_{0}$ of the geodesic probe for different Gauss-Bonnet coefficient $\alpha$ and different charge $Q$ at $v_{\star}=-0.856,-0.456$ respectively.

velocity, which is plotted in figure 6 . From this figure, we can observe two interesting phenomena for the thermalization process. One is that the thermalization velocity is negative at the initial time while it is positive at the middle and later periods, which means that the thermalization process is non-monotonic. In fact, this non-monotonic behavior has also been observed in [21]. The reason for this behavior is that at the initial time the thermalization is "quantum" while at the later periods, it is "classical". The author in [21] further argued that at the "quantum" stage the slope of the thermalization curve, namely the thermalization velocity, is negative and at the "classical" stage the slope is positive. Obviously, our result plotted in figure 6 confirms their argument. The other is that there is a phase transition point for the thermalization velocity, which divides the thermalization into an acceleration phase and a deceleration phase. (a) and (b) in figure 6 represent respectively the effect of $Q$ and $\alpha$ on the phase transition points. From both figures, we know that the phase transition points vary for different $Q$ and $\alpha$. Especially in (b), we can observe clearly that as $\alpha$ increases, the phase transition points shift left. At a fixed $Q$ and $\alpha$, we can get the value of the phase transition point. For the case $Q=0.5$ and $\alpha=0.0001$, it is easy to find that in the time range $0<t_{0}<1.04978$, the thermalization is an acceleration process while for $t_{0}>1.04978$ it is a deceleration process before it approaches to the equilibrium state. Surely as the slope of this velocity curve is produced, we also can get the values of the acceleration and deceleration.

Adopting similar strategy, we also can study the motion profile of minimal area as well as the change of the renormalized minimal area surface to thermalization time. Based on the motion equations in (3.24) and the boundary conditions in (3.18), the numerical solution of $z(x)$ can be produced. In this case, we can get the motion profile of minimal area for different charge $Q$ and Gauss-Bonnet coefficient $\alpha$, which is shown in figure 7. From this figure, we know that for a fixed charge, e.g. the first row, as the Gauss-Bonnet coefficient increases, the shell surface approaches to the horizon surface step by step, which means the thermalization is faster. For a fixed Gauss-Bonnet coefficient, e.g. the third column, as the charge increases, the shell surface is removed from the horizon surface step by step, which means the thermalization is slower. The thermalization time for different $\alpha$ and $Q$ have been listed in table (2). It is shown that for a fixed charge the thermalization time decreases as $\alpha$ becomes larger, while for a fixed $\alpha$, the thermalization time increases as $Q$ becomes larger. That is, the charge has an inverse effect compared with the Gauss-Bonnet coefficient on the thermalization time. This phenomenon is similar to that of the geodesics. 


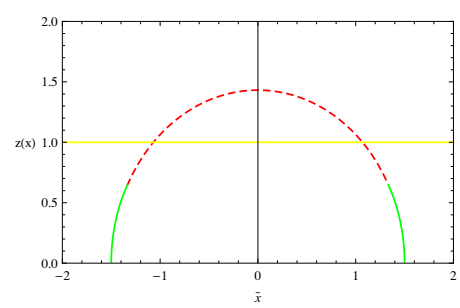

(a) $Q=0.00001, \alpha=-0.1$

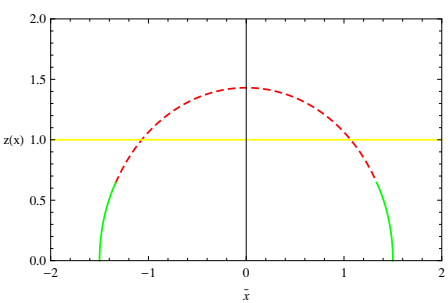

(d) $Q=0.5, \alpha=-0.1$

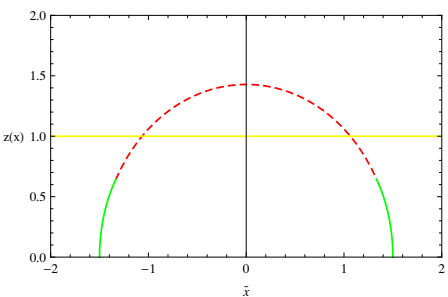

(g) $Q=1, \alpha=-0.1$

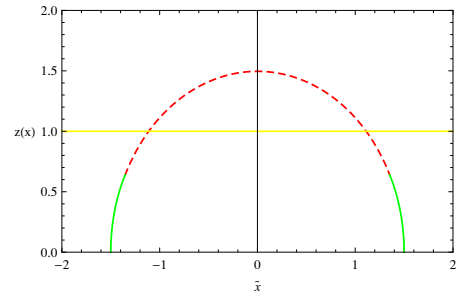

(b) $Q=0.00001, \alpha=0.0001$

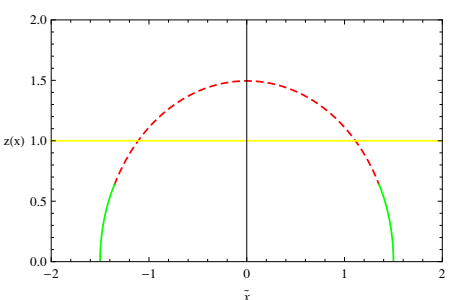

(e) $Q=0.5, \alpha=0.0001$

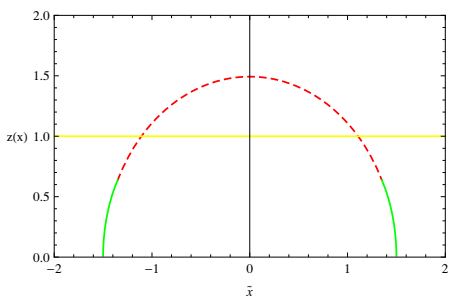

(h) $Q=1, \alpha=0.0001$

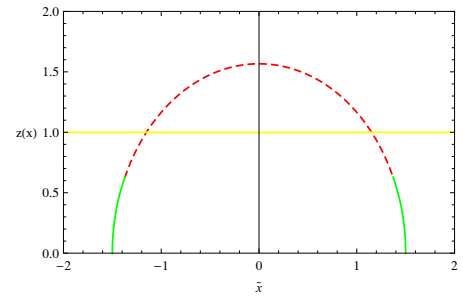

(c) $Q=0.00001, \alpha=0.08$

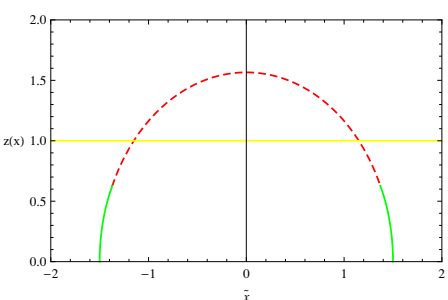

(f) $Q=0.5, \alpha=0.08$

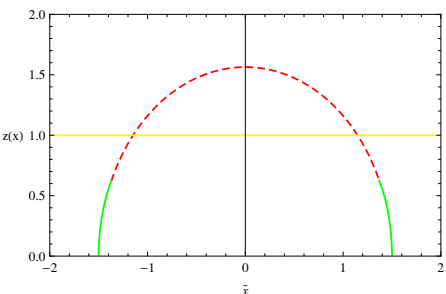

(i) $Q=1, \alpha=0.08$

Figure 1. Motion profile of the geodesics in the charged Gauss-Bonnet Vaidya AdS black brane. The separation of the boundary field theory operator pair is $\tilde{\ell}=3$ and the initial time is $v_{\star}=-0.856$. The black brane horizon is indicated by the yellow line. The position of the shell is described by the junction between the dashed red line and the green line.

\begin{tabular}{|l|c|c|c|}
\hline & $\alpha=-0.1$ & $\alpha=0.0001$ & $\alpha=0.08$ \\
\hline$Q=0.00001$ & 1.01732 & 0.963053 & 0.911401 \\
\hline$Q=0.5$ & 1.02170 & 0.968943 & 0.918953 \\
\hline$Q=1$ & 1.03334 & 0.984877 & 0.939824 \\
\hline
\end{tabular}

Table 2. The thermalization time $t_{0}$ of the geodesic probe for different Gauss-Bonnet coefficient $\alpha$ and different charge $Q$ at a initial time $v_{\star}=-0.252$.

Substituting the numerical result of $z(x)$ into (3.25), we can get the renormalized minimal area surface. Similar to the case of geodesic, we will plot $\delta A-\delta A_{C G B}$, where $\delta A=\delta \tilde{A} / \tilde{l}$ and $\delta A_{C G B}$ is the renormalized minimal area surface for a charged GaussBonnet AdS black brane. The relation between the renormalized minimal area surface and thermalization time for different charge $Q$ is given in figure 8 for a fixed Gauss-Bonnet coefficient $\alpha$, in which the vertical axis indicates the renormalized minimal area surface while the horizontal axis indicates the thermalization time $t_{0}$. For a fixed $\alpha$, we find larger 


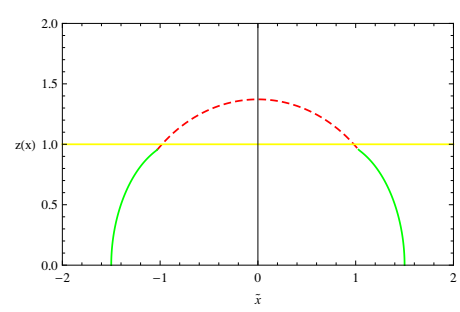

(a) $Q=0.00001, \alpha=-0.1$

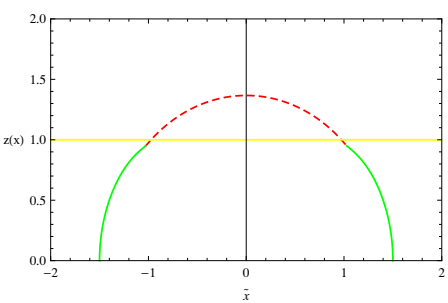

(d) $Q=0.5, \alpha=-0.1$

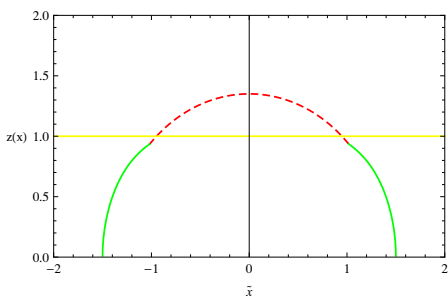

(g) $Q=1, \alpha=-0.1$

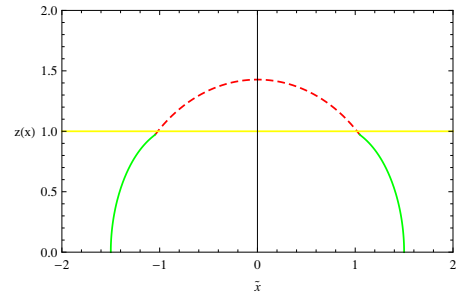

(b) $Q=0.00001, \alpha=0.0001$

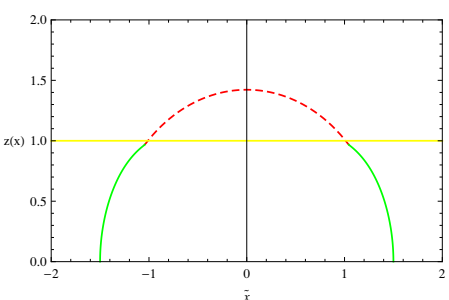

(e) $Q=0.5, \alpha=0.0001$

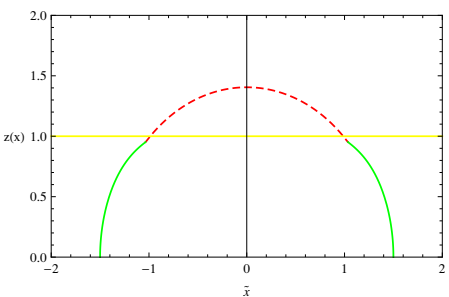

(h) $Q=1, \alpha=0.0001$

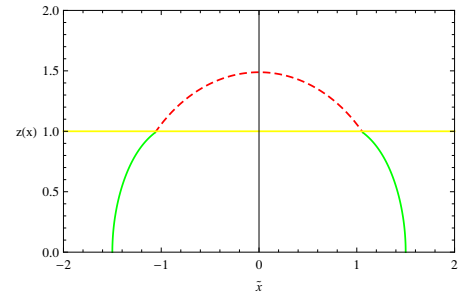

(c) $Q=0.00001, \alpha=0.08$

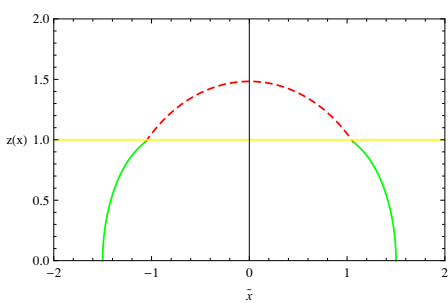

(f) $Q=0.5, \alpha=0.08$

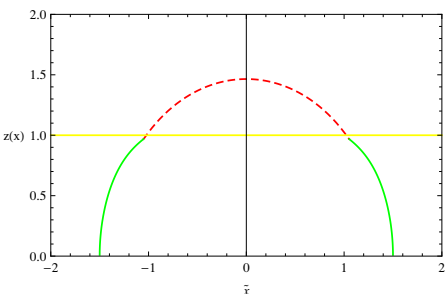

(i) $Q=1, \alpha=0.08$

Figure 2. Motion profile of the geodesics in the charged Gauss-Bonnet Vaidya AdS black brane. The separation of the boundary field theory operator pair is $\tilde{\ell}=3$ and the initial time is $v_{\star}=-0.456$. The black brane horizon is indicated by the yellow line. The position of the shell is described by the junction between the dashed red line and the green line.

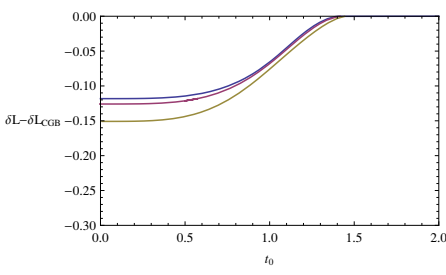

(a) $\alpha=-0.1$

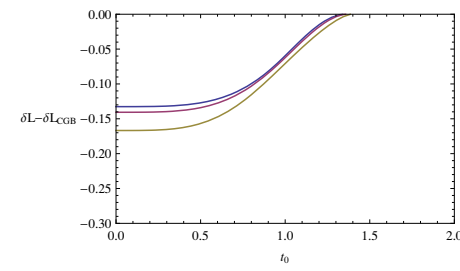

(b) $\alpha=0.0001$

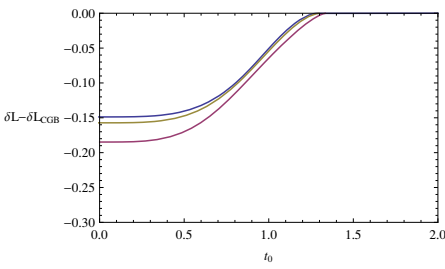

(c) $\alpha=0.08$

Figure 3. Thermalization of the renormalized geodesic lengths in a charged Gauss-Bonnet Vaidya AdS black brane for different charge $Q$ at a fixed Gauss-Bonnet coefficient $\alpha$. The separation of the boundary field theory operator pair is $\tilde{\ell}=3$. The green line, red line and purple line correspond to $Q=0.00001,0.5,1$ respectively.

the charge $Q$ is, longer the thermalization time is. That is to say, as the chemical potential in the dual field theory increases, the thermalization time rises too, which is the same as that obtained by studying the motion profile of the minimal area. In addition, for a fixed charge, we also study the effect of the Gauss-Bonnet coefficient on the thermalization 


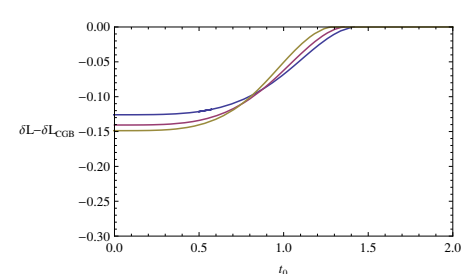

(a) $Q=0.00001$

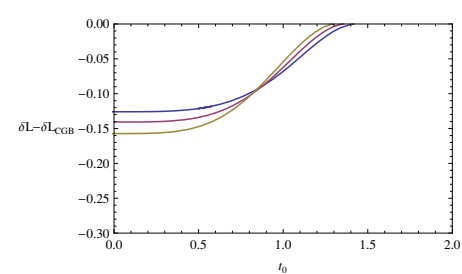

(b) $Q=0.5$

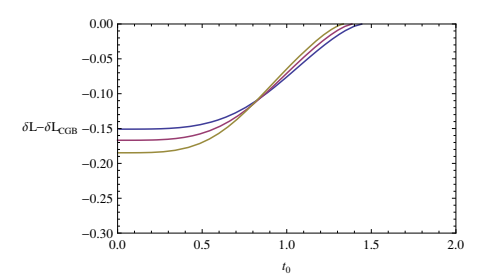

(c) $Q=1$

Figure 4. Thermalization of the renormalized geodesic lengths in a charged Gauss-Bonnet Vaidya AdS black brane for different Gauss-Bonnet coefficients $\alpha$ at a fixed charge $Q$. The separation of the boundary field theory operator pair is $\tilde{\ell}=3$. The green line, red line and purple line correspond to $\alpha=-0.1,0.0001,0.08$ respectively.

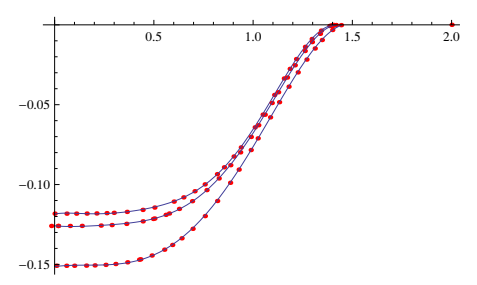

(a) $\alpha=-0.1$

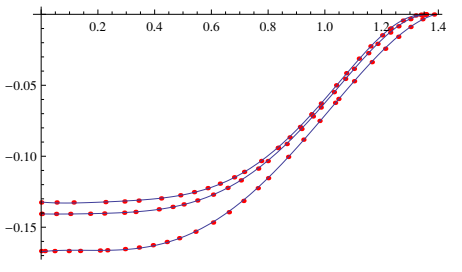

(b) $\alpha=0.0001$

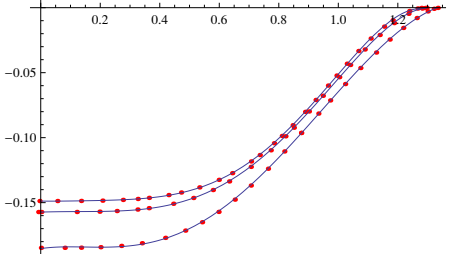

(c) $\alpha=0.08$

Figure 5. Comparison of the function in eq. (4.3) with the numerical result in figure 3.

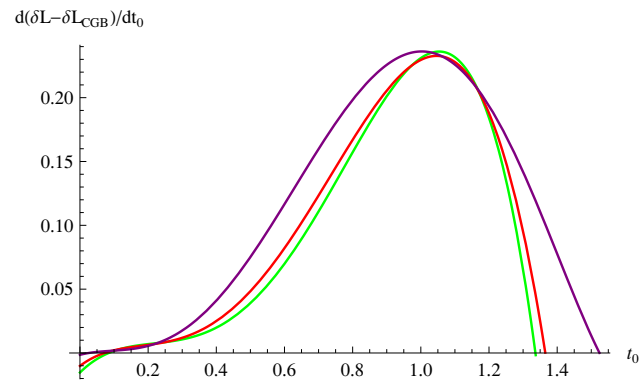

(a) $\alpha=0.0001$ and $\tilde{\ell}=3$

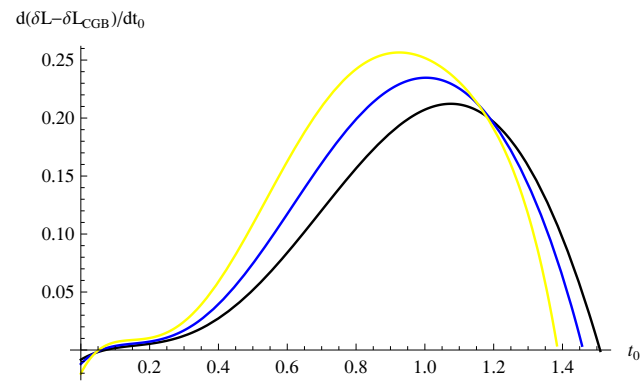

(b) $Q=1$ and $\tilde{\ell}=3$

Figure 6. Thermalization velocity of the renormalized geodesic lengths in a charged Gauss-Bonnet Vaidya AdS black brane. The green line, red line and purple line in (a) correspond to $Q=$ 0.00001, 0.5, 1 and the black line, blue line and yellow line in (b) correspond to $\alpha=-0.1,0.0001,0.08$ respectively.

time, which is shown in figure 9. It is obvious that larger the Gauss-Bonnet coefficient is, shorter the thermalization time is, which means that the quark gluon plasma is easier to thermalize. This behavior is similar to that of the geodesic which is given in figure 3 . As the case of the renormalized geodesic length, we find there is also an overlapped region for the case $Q=0.00001,0.5,1$ respectively in figure 9. We also can get the functions of the renormalized minimal area surface with respect to the thermalization time. At $\alpha=0.08$, the functions of the thermalization curve for $Q=0.00001,0.5,1$ can be expressed 


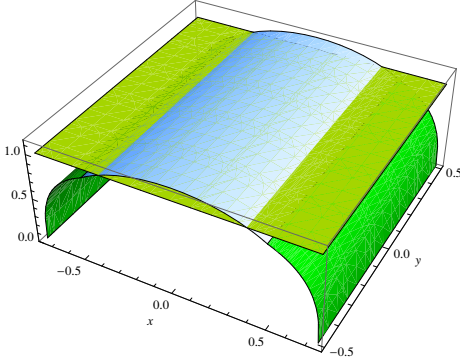

(a) $Q=0.00001, \alpha=-0.1$

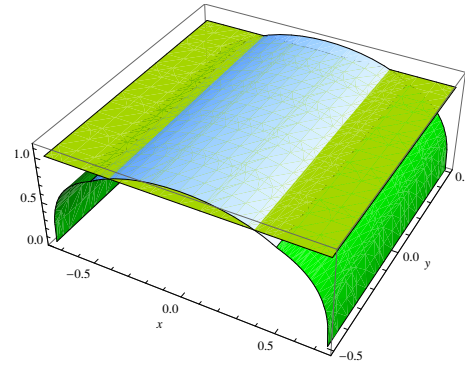

(d) $Q=0.5, \alpha=-0.1$

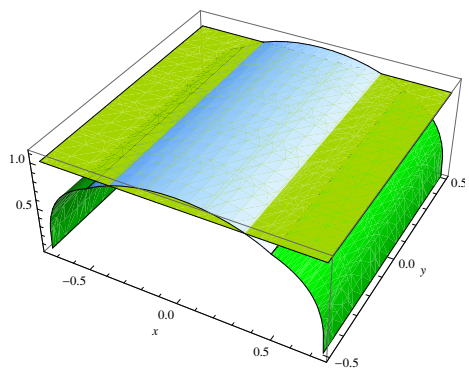

(g) $Q=1, \alpha=-0.1$

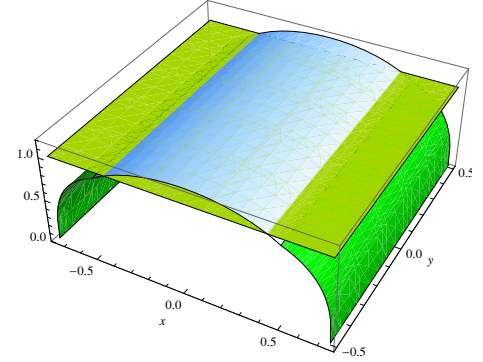

(b) $Q=0.00001, \alpha=0.0001$

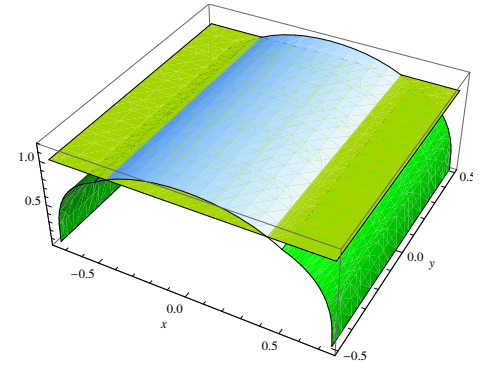

(e) $Q=0.5, \alpha=0.0001$

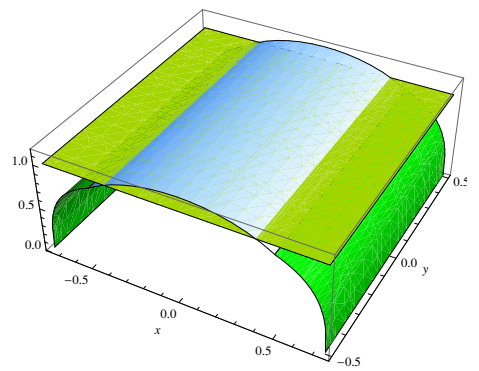

(h) $Q=1, \alpha=0.0001$

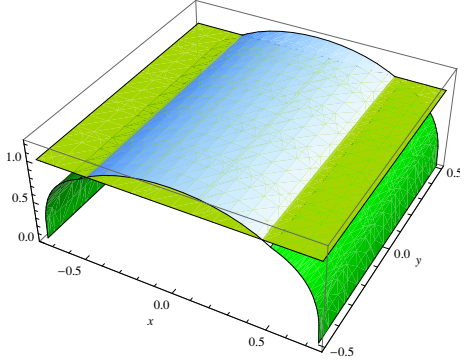

(c) $Q=0.00001, \alpha=0.08$

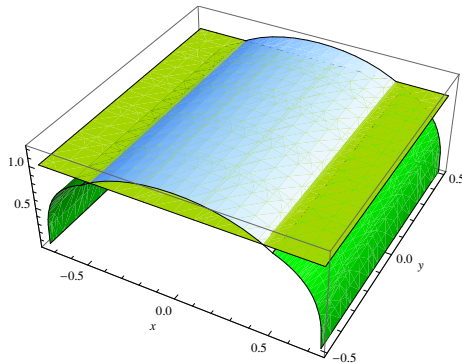

(f) $Q=0.5, \alpha=0.08$

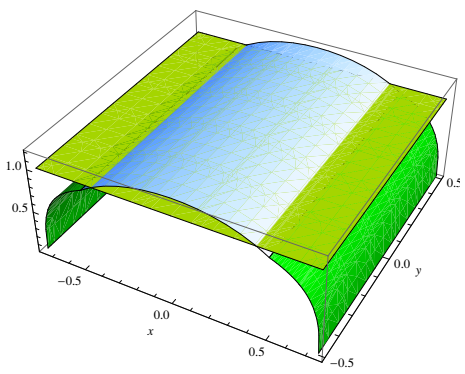

(i) $Q=1, \alpha=0.08$

Figure 7. Motion profile of the minimal area in the charged Gauss-Bonnet Vaidya AdS black brane. The boundary separation along the $x$ direction is 1.5 , and along the $y$ direction is 1 , the initial time is $v_{*}=-0.252$. The yellow surface is the location of the horizon. The position of the shell is described by the junction between the white surface and the green surface.

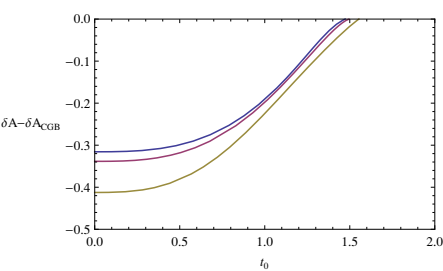

(a) $\alpha=-0.1$

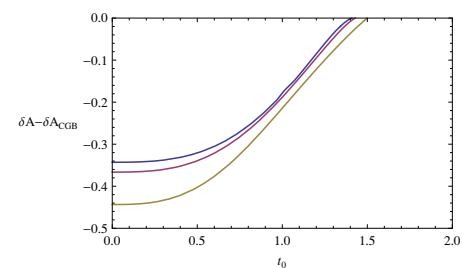

(b) $\alpha=0.0001$

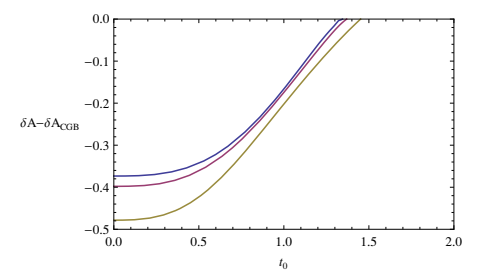

(c) $\alpha=0.08$

Figure 8. Thermalization of the renormalized minimal surface area in a charged Gauss-Bonnet Vaidya AdS black brane for different charge $Q$ at a fixed Gauss-Bonnet coefficients $\alpha$. The separation of the boundary field theory operator pair is $\tilde{\ell}=2$. The green line, red line and purple line correspond to $Q=0.00001,0.5,1$ respectively. 


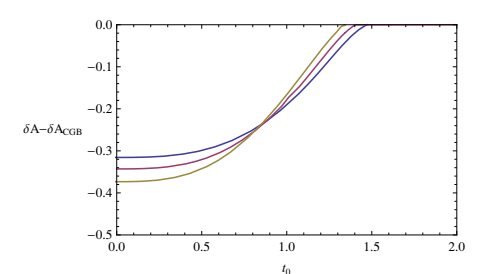

(a) $Q=0.00001$

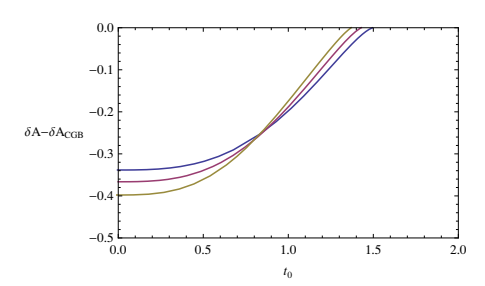

(b) $Q=0.5$

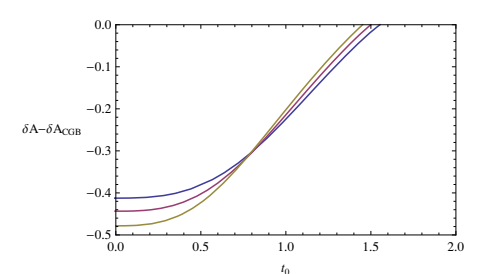

(c) $Q=1$

Figure 9. Thermalization of the renormalized minimal surface area in a charged Gauss-Bonnet Vaidya AdS black brane for different Gauss-Bonnet coefficients $\alpha$ at a fixed charge $Q$. The separation of the boundary field theory operator pair is $\tilde{\ell}=2$. The green line, red line and purple line correspond to $\alpha=-0.1,0.0001,0.08$ respectively.

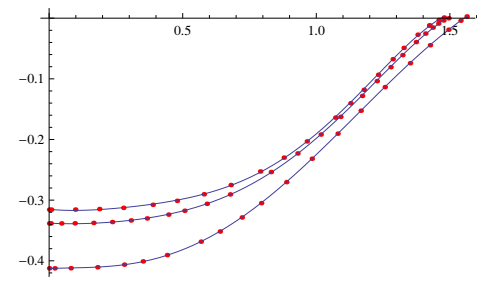

(a) $\alpha=-0.1$

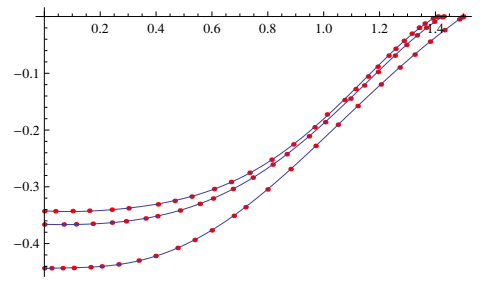

(b) $\alpha=0.0001$

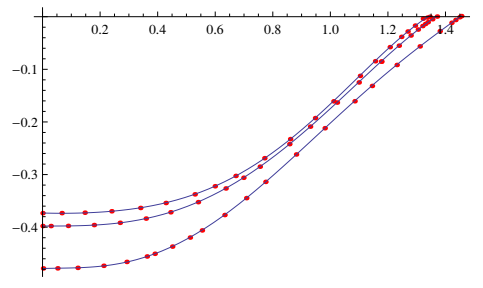

(c) $\alpha=0.08$

Figure 10. Comparison of the function in eq. (4.4) with the numerical result in figure 8.

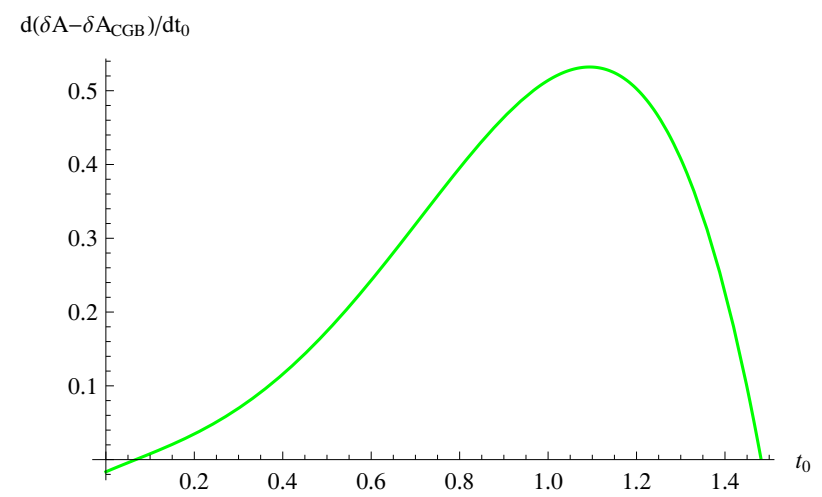

Figure 11. Thermalization velocity of the renormalized minimal surface area in a charged GaussBonnet Vaidya AdS black brane for $Q=0.00001$ and $\alpha=0.08$.

respectively as

$$
\left\{\begin{aligned}
h_{1}=-0.373032- & 0.0165426 t_{0}+0.128311 t_{0}^{2} \\
& -0.0886585 t_{0}^{3}+0.379981 t_{0}^{4}-0.19601 t_{0}^{5} \\
h_{2}=-0.397816+ & 0.000718227 t_{0}-0.0130504 t_{0}^{2} \\
+ & 0.383351 t_{0}^{3}-0.115431 t_{0}^{4}-0.0324124 t_{0}^{5} \\
h_{3}=-0.478534+ & 0.0172206 t_{0}-0.165658 t_{0}^{2} \\
+ & 1.09677 t_{0}^{3}-0.887269 t_{0}^{4}+0.215318 t_{0}^{5}
\end{aligned}\right.
$$

That is, the thermalization curve can be described by a function of time $t_{0}$ with different 
modulus for different charges and different Gauss-Bonnet coefficients. With the thermalization curve, we further can get the thermalization velocity. For the case $\alpha=0.08$ and $Q=0.00001$, the thermalization velocity is plotted in figure 11. From this figure, we also can observe the non-monotonic behavior and phase transition point of the thermalization process, which is similar to that of the renormalized geodesic length. We also can obtain the acceleration phase and deceleration phase for this case. By the slope of the thermalization velocity, we know that the acceleration range is $0<t_{0}<1.106$ and the deceleration range is $1.106<t_{0}<1.481$. Adopting the same strategy, we also can get the phase transition points for other $Q$ and $\alpha$.

\section{Conclusions}

Effect of the chemical potential and correction parameter on the thermalization in the dual boundary field theory is investigated by considering the collapse of a shell of charged dust that interpolates between a pure AdS and a charged Gauss-Bonnet AdS black brane. The two-point function and expectation values of Wilson loop are chosen as the thermalization probes, which are dual to the renormalized geodesic length and minimal area surface in the bulk. We first study the motion profiles of the geodesic and minimal surface and find that larger the Gauss-Bonnet coefficient is, shorter the thermalization time is, and larger the charge is, longer the thermalization time is. At the initial stage of the thermalization, we find that the charge has little effect on the thermalization time. We reproduce this result by studying the relation between the renormalized geodesic length and time as well as the renormalized minimal surface area and time respectively.

In addition, we also find the functions of the thermalization probes with respect to the thermalization time by fitting the numerical result. Though this is a naive test, we still can get some useful information. Firstly, we get the thermalization velocity for a fixed charge and Gauss-Bonnet coefficient. From the velocity curve, on one hand, we know that the thermalization process is non-monotonic, and on the other hand we find there is a phase transition point, which divides the thermalization into an acceleration phase and a deceleration phase. Secondly according to the slope of the velocity curve, we also get the acceleration and deceleration of the thermalization. Thus our investigation provides a more accurate description of the thermalization process. Note that in our naive test, we can not see how the charge and Gauss-Bonnet coefficient affect the thermalization quantificationally since the coefficients of the functions are not determined. In future, we expect to find an analytical formulism to study the thermalization so that we can get more useful information on the thermalization process.

\section{Acknowledgments}

We would like to give great thanks to the anonymous referee for his helpful suggestions about this paper. Xiao-Xiong Zeng would like to thank Hongbao Zhang for his encouragement and various valuable suggestions during this work. This work is supported in part by the National Natural Science Foundation of China (Grant Nos. 11365008, 61364030). It is 
also supported by the Natural Science Fund of Education Department of Hubei Province (Grant No. Q20131901)

\section{A Derivation of the Hamiltonian from the Lagrangian}

Suppose that there exists a Hamiltonian $H$ such that

$$
U(x, y ; T)=\left\langle x\left|e^{-i H T}\right| y\right\rangle=\mathcal{N} \prod_{\tau} \int d^{d+1} X(\tau) \sqrt{-g(\tau)} e^{i \int_{0}^{T} d \tau L(\tau)}
$$

with $X(0)=y, X(T)=x$, and the Lagrangian $L=\frac{1}{2}\left(g_{\mu \nu} \frac{d X^{\mu}}{d \tau} \frac{d X^{\nu}}{d \tau}-m^{2}\right)$. Then we have

$$
i \frac{\partial}{\partial T} U(x, y ; T)=H U(x, y ; T),
$$

and

$$
\begin{aligned}
U(x, y ; T)=N \int d^{d+1} X \sqrt{-g(X)} \\
\\
e^{\frac{i}{2 \epsilon} g_{\mu \nu}\left(\frac{x+X}{2}\right)(x-X)^{\mu}(x-X)^{\nu}-\frac{i}{2} \epsilon m^{2}} U(X, y ; T-\epsilon),
\end{aligned}
$$

where $\epsilon$ is a small quantity, to be taken to go to zero in the later calculation. Now for convenience, we would like to resort to the Rienmann normal coordinate at $x$, where the behavior of metric is simplified as

$$
g_{\mu \nu}(x)=\eta_{\mu \nu}, \partial_{\rho} g_{\mu \nu}(x)=0, \partial_{\rho} \partial_{\sigma} g_{\mu \nu}(x)=-\frac{1}{3}\left[R_{\mu \rho \nu \sigma}(x)+R_{\mu \sigma \nu \rho}(x)\right] .
$$

So by Taylor expanding all the involved functions at $x$ and $T$, eq. (A.3) gives rise to

$$
\begin{array}{rl}
U(x, y ; T)=N \int d^{d+1} & X \sqrt{-g(x)}\left[1-\frac{1}{6} R_{\rho \sigma}(x)(x-X)^{\rho}(x-X)^{\sigma}+\cdots\right] \\
& e^{\frac{i}{2 \epsilon} g_{\mu \nu}(x)(x-X)^{\mu}(x-X)^{\nu}}\left(1-\frac{i}{2} \epsilon m^{2}+\cdots\right) \\
& {\left[1-(x-X)^{\rho} \partial_{\rho}+\frac{1}{2}(x-X)^{\rho}(x-X)^{\sigma} \partial_{\rho} \partial_{\sigma}+\cdots\right]} \\
& \left(1-\epsilon \frac{\partial}{\partial T}+\cdots\right) U(x, y ; T) .
\end{array}
$$

Here we only keep those terms up to the first order of $\epsilon$ after the Gaussian integral, where the normalization constant $N$ can be fixed by the zero order equation and $H$ can be determined by the first order equation as

$$
H=-\frac{1}{2}\left[\nabla^{a} \nabla_{a}-m^{2}\right]+\frac{1}{6} R .
$$

Similarly, if the Lagrangian is given by

$$
L=\frac{1}{2}\left(g_{\mu \nu} \frac{d X^{\mu}}{d \tau} \frac{d X^{\nu}}{d \tau}-m^{2}\right)+\frac{1}{6} R,
$$

then the corresponding Hamiltonian will be shifted to

$$
H=-\frac{1}{2}\left[\nabla^{a} \nabla_{a}-m^{2}\right] .
$$


Open Access. This article is distributed under the terms of the Creative Commons Attribution License (CC-BY 4.0), which permits any use, distribution and reproduction in any medium, provided the original author(s) and source are credited.

\section{References}

[1] J.M. Maldacena, The large- $N$ limit of superconformal field theories and supergravity, Adv. Theor. Math. Phys. 2 (1998) 231 [Int. J. Theor. Phys. 38 (1999) 1113] [hep-th/9711200] [INSPIRE].

[2] J. Sonner and A.G. Green, Hawking radiation and non-equilibrium quantum critical current noise, Phys. Rev. Lett. 109 (2012) 091601 [arXiv: 1203.4908] [INSPIRE].

[3] W.-J. Li, Y. Tian and H.-b. Zhang, Periodically driven holographic superconductor, JHEP 07 (2013) 030 [arXiv: 1305.1600] [INSPIRE].

[4] K. Murata, S. Kinoshita and N. Tanahashi, Non-equilibrium condensation process in a holographic superconductor, JHEP 07 (2010) 050 [arXiv: 1005.0633] [INSPIRE].

[5] A. Mukhopadhyay, Nonequilibrium fluctuation-dissipation relation from holography, Phys. Rev. D 87 (2013) 066004 [arXiv:1206.3311] [INSPIRE].

[6] A. Kundu and S. Kundu, Steady-state physics, effective temperature dynamics in holography, arXiv:1307.6607 [INSPIRE].

[7] S. Nakamura, Nonequilibrium phase transitions and nonequilibrium critical point from AdS/CFT, Phys. Rev. Lett. 109 (2012) 120602 [arXiv:1204.1971] [INSPIRE].

[8] R. Baier, A.H. Mueller, D. Schiff and D. Son, 'Bottom up' thermalization in heavy ion collisions, Phys. Lett. B 502 (2001) 51 [hep-ph/0009237] [INSPIRE].

[9] D. Garfinkle and L.A. Pando Zayas, Rapid thermalization in field theory from gravitational collapse, Phys. Rev. D 84 (2011) 066006 [arXiv:1106.2339] [INSPIRE].

[10] D. Garfinkle, L.A. Pando Zayas and D. Reichmann, On field theory thermalization from gravitational collapse, JHEP 02 (2012) 119 [arXiv:1110.5823] [INSPIRE].

[11] A. Allais and E. Tonni, Holographic evolution of the mutual information, JHEP 01 (2012) 102 [arXiv:1110.1607] [INSPIRE].

[12] S.R. Das, Holographic quantum quench, J. Phys. Conf. Ser. 343 (2012) 012027 [arXiv:1111.7275] [INSPIRE].

[13] D. Steineder, S.A. Stricker and A. Vuorinen, Probing the pattern of holographic thermalization with photons, JHEP 07 (2013) 014 [arXiv: 1304.3404] [INSPIRE].

[14] B. Wu, On holographic thermalization and gravitational collapse of massless scalar fields, JHEP 10 (2012) 133 [arXiv:1208.1393] [INSPIRE].

[15] X. Gao, A.M. Garcia-Garcia, H.B. Zeng and H.-Q. Zhang, Lack of thermalization in holographic superconductivity, arXiv:1212.1049 [INSPIRE].

[16] A. Buchel, L. Lehner, R.C. Myers and A. van Niekerk, Quantum quenches of holographic plasmas, JHEP 05 (2013) 067 [arXiv: 1302.2924] [INSPIRE].

[17] V. Keranen, E. Keski-Vakkuri and L. Thorlacius, Thermalization and entanglement following a non-relativistic holographic quench, Phys. Rev. D 85 (2012) 026005 [arXiv:1110.5035] [INSPIRE]. 
[18] V. Balasubramanian et al., Thermalization of strongly coupled field theories, Phys. Rev. Lett. 106 (2011) 191601 [arXiv:1012.4753] [INSPIRE].

[19] V. Balasubramanian et al., Holographic thermalization, Phys. Rev. D 84 (2011) 026010 [arXiv:1103.2683] [INSPIRE].

[20] D. Galante and M. Schvellinger, Thermalization with a chemical potential from AdS spaces, JHEP 07 (2012) 096 [arXiv:1205.1548] [INSPIRE].

[21] E. Caceres and A. Kundu, Holographic thermalization with chemical potential, JHEP 09 (2012) 055 [arXiv: 1205. 2354] [INSPIRE].

[22] E. Caceres, A. Kundu and D.-L. Yang, Jet quenching and holographic thermalization with a chemical potential, arXiv:1212.5728 [INSPIRE].

[23] X. Zeng and W. Liu, Holographic thermalization in Gauss-Bonnet gravity, Phys. Lett. B 726 (2013) 481 [arXiv:1305.4841] [INSPIRE].

[24] W.H. Baron and M. Schvellinger, Quantum corrections to dynamical holographic thermalization: entanglement entropy and other non-local observables, JHEP 08 (2013) 035 [arXiv:1305.2237] [INSPIRE].

[25] Y.-Z. Li, S.-F. Wu and G.-H. Yang, Gauss-Bonnet correction to Holographic thermalization: two-point functions, circular Wilson loops and entanglement entropy, Phys. Rev. D 88 (2013) 086006 [arXiv:1309.3764] [InSPIRE].

[26] W. Baron, D. Galante and M. Schvellinger, Dynamics of holographic thermalization, JHEP 03 (2013) 070 [arXiv:1212.5234] [InSPIRE].

[27] I. Aref'eva, A. Bagrov and A.S. Koshelev, Holographic thermalization from Kerr-AdS, JHEP 07 (2013) 170 [arXiv:1305.3267] [INSPIRE].

[28] V.E. Hubeny, M. Rangamani and E. Tonni, Thermalization of causal holographic information, JHEP 05 (2013) 136 [arXiv: 1302.0853] [INSPIRE].

[29] I.Y. Arefeva and I.V. Volovich, On holographic thermalization and dethermalization of quark-gluon plasma, arXiv:1211.6041 [INSPIRE].

[30] V. Balasubramanian et al., Thermalization of the spectral function in strongly coupled two dimensional conformal field theories, JHEP 04 (2013) 069 [arXiv:1212.6066] [INSPIRE].

[31] V. Balasubramanian et al., Inhomogeneous holographic thermalization, JHEP 10 (2013) 082 [arXiv:1307.7086] [INSPIRE].

[32] V. Balasubramanian et al., Inhomogeneous thermalization in strongly coupled field theories, Phys. Rev. Lett. 111 (2013) 231602 [arXiv: 1307.1487] [INSPIRE].

[33] P. Candelas, G.T. Horowitz, A. Strominger and E. Witten, Vacuum configurations for superstrings, Nucl. Phys. B 258 (1985) 46.

[34] S. Nojiri and S.D. Odintsov, Brane-world cosmology in higher derivative gravity or warped compactification in the next-to-leading order of AdS/CFT correspondence, JHEP 07 (2000) 049.

[35] J. de Boer, M. Kulaxizi and A. Parnachev, Holographic entanglement entropy in Lovelock gravities, JHEP 07 (2011) 109 [arXiv:1101.5781] [INSPIRE].

[36] L.-Y. Hung, R.C. Myers and M. Smolkin, On holographic entanglement entropy and higher curvature gravity, JHEP 04 (2011) 025 [arXiv: 1101.5813] [INSPIRE]. 
[37] A. Bhattacharyya, A. Kaviraj and A. Sinha, Entanglement entropy in higher derivative holography, JHEP 08 (2013) 012 [arXiv:1305.6694] [INSPIRE].

[38] X. Dong, Holographic entanglement entropy for general higher derivative gravity, JHEP 01 (2014) 044 [arXiv:1310.5713] [inSPIRE].

[39] Y.-Z. Li, S.-F. Wu, Y.-Q. Wang and G.-H. Yang, Linear growth of entanglement entropy in holographic thermalization captured by horizon interiors and mutual information, JHEP 09 (2013) 057 [arXiv: 1306.0210] [INSPIRE].

[40] W.-z. Guo, S. He and J. Tao, Note on entanglement temperature for low thermal excited states in higher derivative gravity, JHEP 08 (2013) 050 [arXiv:1305.2682] [INSPIRE].

[41] A. Bhattacharyya, M. Sharma and A. Sinha, On generalized gravitational entropy, squashed cones and holography, JHEP 01 (2014) 021 [arXiv: 1308.5748] [INSPIRE].

[42] R.C. Myers, S. Sachdev and A.Singh, Holographic quantum critical transport without self-duality, Phys. Rev. D 83 (2011) 066017 [arXiv:1010.0443] [INSPIRE].

[43] D. Anninos and G. Pastras, Thermodynamics of the Maxwell-Gauss-Bonnet Anti-de Sitter black hole with higher derivative gauge corrections, JHEP 07 (2009) 030 [arXiv: 0807.3478] [INSPIRE].

[44] R.-G. Cai, Gauss-Bonnet black holes in AdS spaces, Phys. Rev. D 65 (2002) 084014 [hep-th/0109133] [INSPIRE].

[45] S. Cremonini, K. Hanaki, J.T. Liu and P. Szepietowski, Black holes in five-dimensional gauged supergravity with higher derivatives, JHEP 12 (2009) 045 [arXiv:0812.3572] [INSPIRE].

[46] D. Astefanesei, N. Banerjee and S. Dutta, (Un)attractor black holes in higher derivative AdS gravity, JHEP 11 (2008) 070 [arXiv:0806.1334] [INSPIRE].

[47] X.O. Camanho and J.D. Edelstein, Causality constraints in AdS/CFT from conformal collider physics and Gauss-Bonnet gravity, JHEP 04 (2010) 007 [arXiv:0911.3160] [INSPIRE].

[48] X.O. Camanho and J.D. Edelstein, Causality in AdS/CFT and Lovelock theory, JHEP 06 (2010) 099 [arXiv:0912.1944] [INSPIRE].

[49] A. Buchel et al., Holographic GB gravity in arbitrary dimensions, JHEP 03 (2010) 111 [arXiv:0911.4257] [INSPIRE].

[50] X.-H. Ge, Y. Ling, Y. Tian and X.-N. Wu, Holographic RG flows and transport coefficients in Einstein-Gauss-Bonnet-Maxwell theory, JHEP 01 (2012) 117 [arXiv:1112.0627] [INSPIRE].

[51] A.E. Dominguez and E. Gallo, Radiating black hole solutions in Einstein-Gauss-Bonnet gravity, Phys. Rev. D 73 (2006) 064018 [gr-qc/0512150] [INSPIRE].

[52] T. Kobayashi, A Vaidya-type radiating solution in Einstein-Gauss-Bonnet gravity and its application to braneworld, Gen. Rel. Grav. 37 (2005) 1869 [gr-qc/0504027] [INSPIRE].

[53] H. Maeda, Effects of Gauss-Bonnet terms on final fate of gravitational collapse, Class. Quant. Grav. 23 (2006) 2155 [gr-qc/0504028] [INSPIRE].

[54] E. Caceres, A. Kundu, J.F. Pedraza and W. Tangarife, Strong subadditivity, null energy condition and charged black holes, JHEP 01 (2014) 084 [arXiv: 1304.3398] [INSPIRE]. 
[55] V. Balasubramanian and S.F. Ross, Holographic particle detection, Phys. Rev. D 61 (2000) 044007 [hep-th/9906226] [INSPIRE].

[56] J.M. Maldacena, Wilson loops in large- $N$ field theories, Phys. Rev. Lett. 80 (1998) 4859 [hep-th/9803002] [INSPIRE].

[57] R.C. Myers, M.F. Paulos and A. Sinha, Holographic hydrodynamics with a chemical potential, JHEP 06 (2009) 006 [arXiv:0903.2834] [INSPIRE].

[58] Y. Ling, C. Niu, J.-P. Wu and Z.-Y. Xian, Holographic lattice in Einstein-Maxwell-dilaton gravity, JHEP 11 (2013) 006 [arXiv: 1309.4580] [INSPIRE]. 